\title{
Roundabout Design Research Based on Cellular Automata Simulation
}

\author{
Huangjing Zhang \\ School of Energy \& Power Engineering, North China Electric Power University, Baoding 071000, \\ China.
}

784314165@qq.com

\begin{abstract}
Nowadays, with the development of society and the progress of material life, ownership of vehicles is on the increase day by day. In order to solve the problem of traffic jam, I design some solutions of roundabouts. For practical purposes and universality, to begin with, I choose conventional non-controlled roundabout with single line to research. The whole model is divided into three submodels. Submodel 1 is a dynamic programming model. According to Wardrop Equation, I get the relationship between roundabout's geometric parameter and maximum traffic volume. The submodel 2 is a roundabout model based on Cellular Automata. With the help of submodel 1, I design a roundabout with optimal geometrical parameter. I assume that the vehicle's arrival obey Poisson distribution with mean $\lambda$, and I use the value of $\lambda$ to represent the number of cars. The total delay time is used to measure the total number of stopping vehicles, which is similar to characterize the performance of the whole roundabout system. Through the research above, I can draw up a conclusion: It is suitable for a smooth road while intersection with signal lights is suitable for a heavy traffic road. As for this model, its innovation is that I bring in Cellular Automaton to make simulation. Therefore, our model is powerful and reliable for various types of different roundabouts' design.
\end{abstract}

Keywords: Roundabout; Wardrop Equation; Cellular Automaton; Poisson distribution.

\section{Restatement of the Problem}

Helping design a roundabout is a problem which can be split into two parts: designing its geometry, and optimizing the roundabout traffic capacity. I concluded that I should be able to use the Wardrop Equation which widely applies to the roundabout traffic to analyze the geometry of the roundabout. Furthermore, I realized that the traffic capacity is closely affected by how many seconds each light should remain green, how many traffic lanes should be set and so on

\section{Classification of the Roundabout}

(1) Regular circle

This is the most common type in the traffic design around the world. In the meanwhile, it is usually used to design an intersection with a long mixed section and import channels with the same width, while the diameter of the intersection is above $25 \mathrm{~m}$.

(2) Small circle

The diameter of this circle is usually under $25 \mathrm{~m}$. And it's convenient for vehicles to broaden the channels when they get into or get out of the circle.

(3) Tiny rotary

The central area of the roundabout may be other shapes and the radius of the central circle is less than $4 \mathrm{~m}$ (Fig. 1). 


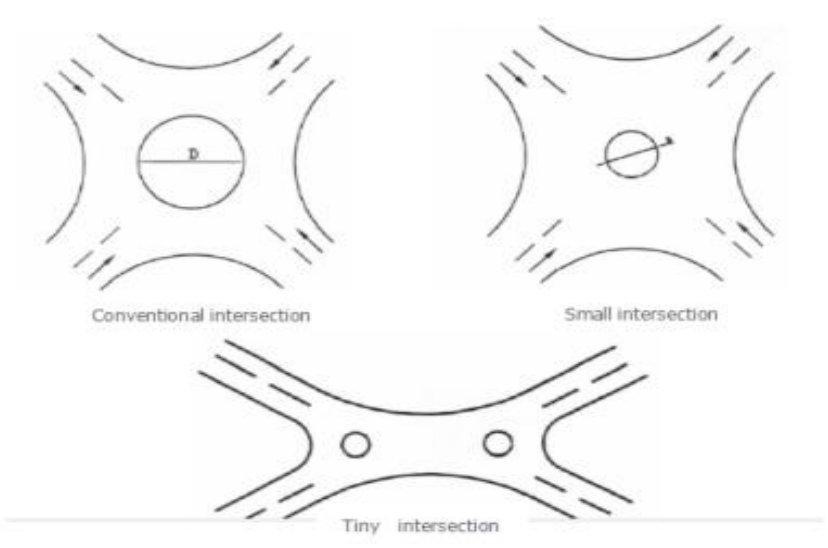

Fig. 1 types of roundabout

\subsection{Classifying Roundabouts by the Number of Import Lanes.}

(1) roundabout with multiple lanes

(2) roundabout with one lane

\section{Assumptions}

The assumptions are shown in Table 1.

Table 1

\begin{tabular}{cl}
\hline Parameter & \multicolumn{1}{c}{ Definition } \\
\hline$W$ & the shortest distance between the inner circle and weaving section \\
$l$ & length of waving section \\
$e_{1}$ & the shortest distance between the inner circle and the entrance \\
$e_{2}$ & the shortest distance between the entrance to the triangle and the \\
& peripheral edge of the circle \\
$P$ & the ratio of vehicles in weaving sections and the total number of \\
& vehicles on the island \\
$V_{i}(t)$ & the speed of the ith cell at time $t$ \\
$V_{i+1}(t)$ & the speed of the cell in front of ith cell at time $t$ \\
$X_{i}(t)$ & position of vehicle $i$ at time $t$ \\
$V_{i}(t)$ & speed of vehicle $i$ at time $t$ \\
$X_{\text {Stopline }}$ & position of stop lines \\
gapis & distance between vehicle $i$ and vehicle $i+1$ at time $t$ \\
state $(i)$ & directions of vehicle $i .1$ means go straight. 2 means turn right. \\
& 3 means turn left. 4 means turn around. \\
\hline
\end{tabular}

\section{Modeling}

\subsection{Roundabout Design Model.}

Based on the island's biggest capacity, I adopt the famous Wardrop Equation, which is widely applied to conventional roundabout design.

$\max Q_{M}=\frac{280 W\left(1+\frac{e}{w}\right)\left(1-\frac{P}{3}\right)}{1+\frac{W}{l}}$

Obviously, the mixed period of maximum capacity is associated with the design parameters. Through changing parameters, I can obtain the maximum capacity of the roundabout. I adopt Lingo software to solve the problem.

\subsection{Cellular Automata Simulation Model.}

Rule 1: entering the island

Vehicles entering into the road intersections obey Possion Distribution: 
$P(X=k)=\frac{\lambda^{k} e^{-\lambda}}{k !} \quad k=0,1,2 \ldots$

Rule 2:leaving the island

Supposing the number of exports is $\mathrm{N}$ around the roundabout. Otherwise, the possibility of leaving each exit is seemed to be the same.

$P_{1}=P_{2}=\ldots=P_{N}=\frac{1}{N}$

Rule 3:slowing down or stop

If the speed of the current cell is greater than the sum of cell and the grid in front of it, then the car will slow down or even stop.

$V_{i}(t)>V_{i+1}(t)+g a p_{i}(t)$

$V_{i}(t)>\operatorname{gap}_{i}(t)-1$

Rule 4:accelerating

If the speed of the current cell is sloIr than the front grid substracting one, then the cell choose to accelerate.

$V_{i}(t)>\operatorname{gap}_{i}(t)-1$

$V_{i}(t)>V_{i}(t)+1$

Based on all the rules above, I use Matlab to simulate.

\section{Conclusion}

(1) Conventional Single Non-control Circle.

The optimal traffic volume is 7138.279 vehicles per hour. Other parameters are shown in Table 2 and Fig. 2:

Table 2. Parameter of optimal traffic volume

\begin{tabular}{cc}
\hline Parameter & Value \\
\hline Biggest traffic flow & $7138.279 \mathrm{Veh} / \mathrm{h}$ \\
$W$ & $18.000 \mathrm{~m}$ \\
$E$ & $18.000 \mathrm{~m}$ \\
$P$ & 0.400 \\
$e_{1}$ & $9.134328 \mathrm{~m}$ \\
$e_{2}$ & $26.85567 \mathrm{~m}$ \\
$l$ & $80.42020 \mathrm{~m}$ \\
\hline
\end{tabular}

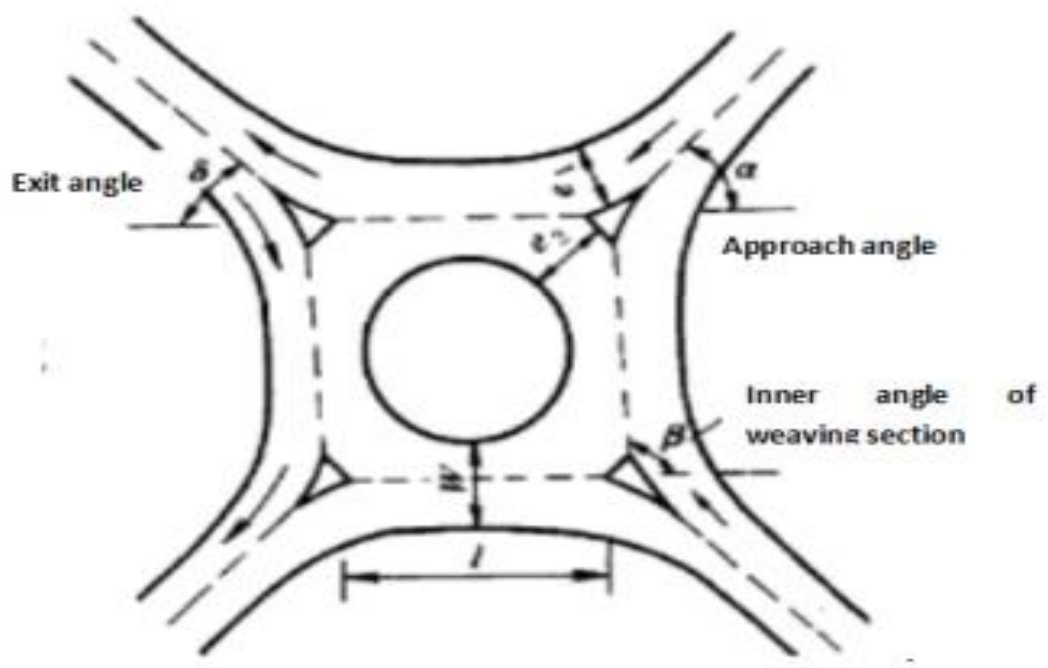

Fig. 2 characteristic of optimal traffic volume 
(2) Light Control Intersection.

This scheme is suitable for large traffic road especially when average headway time $(\lambda)$ is smaller than 3. This scheme is suitable for the two-way two-lane road. The cycle of traffic lights (both red and green) is 6 second. I'll give the schematic in details (Fig. 3).
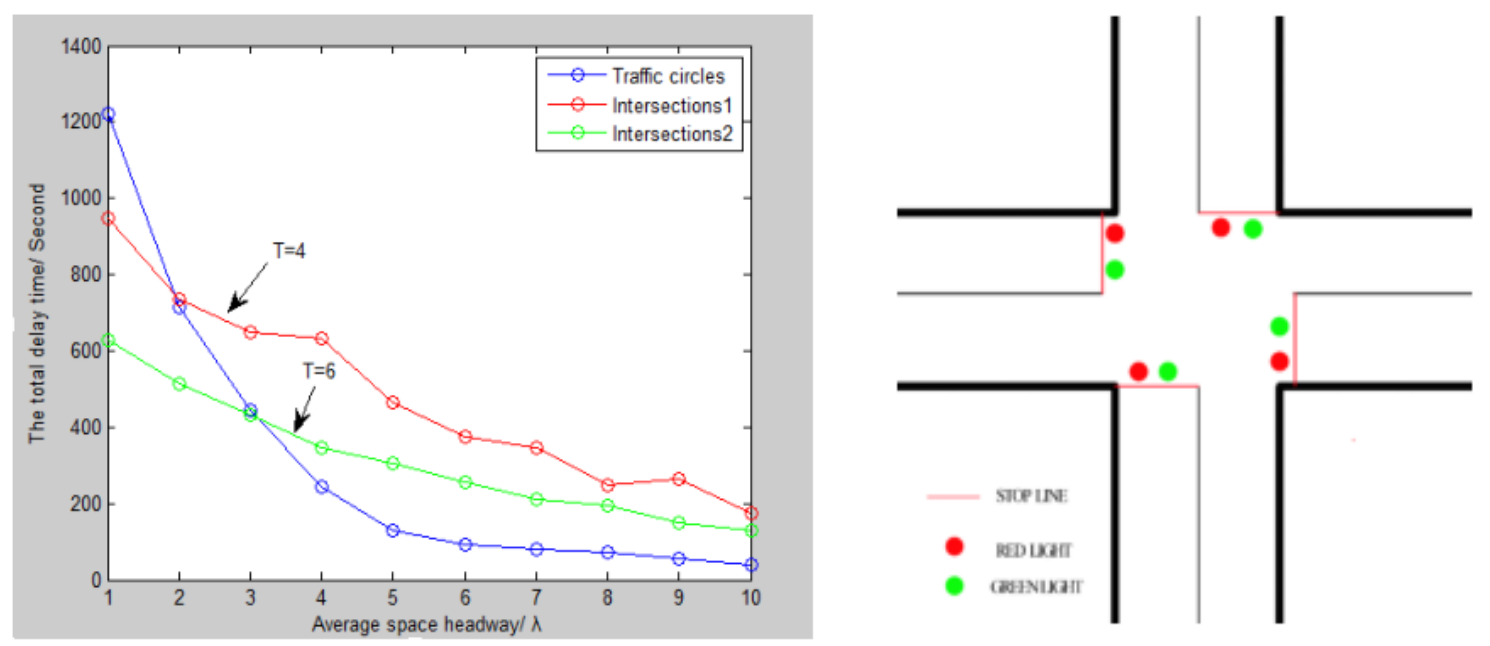

Fig. 3 intersection traffic design

\section{References}

[1] Fenfang Liu. The Study of two-line traffic Flow Characteristics Based on Cellular Automaton [D]. Central South University, 2008.

[2] YiPeng Du. The Theory of Traffic Facilities Designs and Study of Case. South China University of Technology. Central South University, 2013.

[3] Bo Zhu. The Study of Multi-Agents Mixed-Traffic Simulation Based on Cellular Automaton [D]. Shandong University, 2006.

[4] Hailan Zhang. The Analysis of Roundabout's Capacity and the Measures of Improvement [D]. Chang'an University, 2012.

[5] Xiaobao Yang, Ning Zhang. Mathematicl Analysis of Effects of Lanes'Number on Expressway Capacity[J]. Journal of Wuhan University of Technology,2008. 